\title{
Reduced insulin-stimulated GLUT4 bioavailability in stroke-prone spontaneously hypertensive rats
}

Received: 11 August 2004 / Accepted: 29 October 2004 / Published online: 24 February 2005

C) Springer-Verlag 2005

\begin{abstract}
Aims/hypothesis: Insulin-stimulated glucose transport is impaired in a genetic model of hypertension, the stroke-prone spontaneously hypertensive rat (SHRSP), yet the molecular mechanisms that underlie this defect in the animals remain unclear. Methods: We examined the effects of insulin on the trafficking of the insulin-responsive glucose transporter GLUT4 to the plasma membrane in isolated adipocytes from SHRSP and normotensive control WistarKyoto (WKY) rats. Results: Treatment of isolated adipocytes with insulin resulted in trafficking of GLUT4 to the plasma membrane. There was no significant difference in the magnitude of insulin-stimulated GLUT4 trafficking from intracellular membranes to the plasma membrane between strains. In contrast, we demonstrated that there is a significant reduction in GLUT4 accessible to the glucose photolabel Bio-LC-ATB-BGPA at the plasma membrane of SHRSP adipocytes compared with control rats. Conclusions/ interpretation: We propose that a large proportion of GLUT4 translocated to the plasma membrane in response to insulin is not able to bind substrate and catalyse transport in the SHRSP. Therefore, there is a reduction in bioavailable
\end{abstract}

M. Collison · D. J. James · G. W. Gould · I. P. Salt $(\bowtie)$

The Henry Wellcome Laboratory of Cell Biology, Division of Biochemistry and Molecular Biology, Institute of Biomedical and Life Sciences, Davidson Building, University of Glasgow, Glasgow, G12 8QQ, UK

e-mail: i.salt@bio.gla.ac.uk

Tel.: +44-141-3302049

Fax: +44-141-3304620

M. Collison · D. Graham · A. F. Dominiczak

BHF Glasgow Cardiovascular Research Centre, Division of

Cardiovascular and Medical Sciences, University of Glasgow, Glasgow, G11 6NT, UK

\section{G. D. Holman}

Department of Biology and Biochemistry, University of Bath, Bath, BA2 7AY, UK

\section{J. M. C. Connell}

Division of Cardiovascular and Medical Sciences,

Gardiner Institute, Western Infirmary,

Glasgow, G11 6NT, UK
GLUT4 in SHRSP animals that is likely to account, at least in part, for the reduced insulin-stimulated glucose uptake.

Keywords Adipocytes - GLUT4 · Insulin · Photolabel · SHRSP

Abbreviations Bio-LC-ATB-BGPA: 4,4'-O-[2-[2-[2[2-[2-[6-(Biotinylamino)hexanoyl]amino]ethoxy]-ethoxy] ethoxy]-4-(1-azi-2,2,2,-trifluoroethyl)benzoyl]amino-1, 3-propanediyl-bis-D-glucose · IRAP: Insulin-regulated aminopeptidase - KRH: Krebs-Ringer-HEPES buffer LDM: Low-density microsome $\cdot$ PI3K:

Phosphatidylinositol 3'-kinase - PKB: Protein kinase B · PM: Plasma membrane - PMSF: Phenylmethylsulphonyl fluoride - SHRSP: Stroke-prone spontaneously hypertensive rat - VAMP2: Vesicle-associated membrane protein-2 - WKY: Wistar-Kyoto rat

\section{Introduction}

Insulin increases glucose transport activity in muscle and fat cells through the translocation of glucose transporter proteins (primarily GLUT4) from an intracellular location to the plasma membrane. The additional glucose uptake stimulated by insulin is used primarily for the synthesis of glycogen and triacylglycerides in skeletal muscle and adipocytes respectively. The intracellular insulin signalling pathways that lead to GLUT4 translocation have been extensively investigated, yet only the activation of p $85 / \mathrm{p} 110$ phosphatidylinositol 3'-kinase (PI3K) has been identified as an absolute requirement [1].

Reduced insulin-stimulated glucose uptake (insulin resistance) by skeletal muscle and adipose tissue is of major pathogenic importance in a number of human disorders, including type 2 diabetes, hypertension and obesity, yet the underlying molecular mechanisms remain unclear [2]. Insulin resistance is apparent in a genetic model of hypertension, the spontaneously hypertensive rat (SHR) [3, 4], reinforcing the primary nature of the link between car- 
diovascular disorders and insulin resistance. Adipocytes derived from SHR rats exhibit attenuated insulin-stimulated glucose uptake and defective insulin-mediated inhibition of lipolysis compared with control normotensive rats $[3,4]$. We have recently extended these observations in a close relative of the SHR, the stroke-prone spontaneously hypertensive rat (SHRSP). We demonstrated that adipocytes from these animals similarly exhibit attenuated insulin-stimulated glucose uptake and a reduced ability to suppress isoproterenol-stimulated lipolysis [5]. Furthermore, we demonstrated that insulin-stimulated glucose uptake is also attenuated in isolated skeletal muscles of SHRSP rats [6]. However, the mechanisms by which insulin-stimulated glucose transport is reduced in either tissue have not been clarified. No significant difference was demonstrated in the expression or subcellular localisation of IRS-1 and IRS-2, PI3K and GLUT4 in muscles derived from SHRSP compared with in normotensive control WKY rats. In addition, insulin-stimulated protein kinase $\mathrm{B}(\mathrm{PKB})$ activity was also unaltered in muscles from both rats, suggesting insulin-stimulated PI3K activity was not attenuated in the SHRSP [6].

In this study, we investigated the extent of insulinstimulated trafficking of GLUT4 in epididymal adipocytes derived from SHRSP and WKY normotensive control rats. We show that insulin-stimulated trafficking of GLUT4 from intracellular membranes to the plasma membrane is unaltered in adipocytes derived from SHRSP compared with in normotensive WKY control rats as assessed by western blotting subsequent to subcellular fractionation. Despite this apparently unaltered trafficking of GLUT4, we demonstrate a marked attenuation of GLUT4 at the cell surface after stimulation with insulin in adipocytes derived from SHRSP as assessed by accessibility to the photolabel Bio-LC-ATB-BGPA. These data suggest that there is less bioavailable GLUT4 at the plasma membrane of SHRSP adipocytes after insulin stimulation compared with in WKY rats, a phenomenon that may underlie the reduced insulin sensitivity we have previously demonstrated in the SHRSP.

\section{Materials and methods}

Materials Phenylmethylsulphonyl fluoride (PMSF), benzamidine and aprotinin were supplied by Sigma (Poole, Dorset, UK). Horseradish peroxidase-conjugated secondary antibodies were from Amersham Biosciences (Chalfont St. Giles, UK). Insulin was from Novo Nordisk (Copenhagen, Denmark). Type I collagenase was from Worthington (Lakewood, NJ, USA). Thesit was obtained from Roche Diagnostics (Lewes, Sussex, UK). Streptavidin-agarose was from Upstate (Milton Keynes, UK). Mouse anti-IRAP antibodies were kindly supplied by L. Garza and M. Birnbaum (University of Pennsylvania, Philadelphia, PA, USA). The anti-GLUT4 antibody used was obtained as described previously [7]. Anti-VAMP2 antibody was obtained from Synaptic Systems (Göttingen, Germany). Rabbit antip38MAPK and anti-phospho-p38MAPK antibodies were from New England Biolabs (Hitchin, UK). Mouse anti- caveolin antibodies were from BD Biosciences (Oxford, UK). All other reagents were from sources described previously $[5,6]$.

Synthesis of Bio-LC-ATB-BGPA The biotinylated bis(Dglucose) derivative photoaffinity label Bio-LC-ATB-BGPA was synthesised as described previously [8].

Animals Rat strains used in this study were inbred SHRSP and WKY rats obtained from the NIH and maintained in the Division of Cardiovascular and Medical Sciences, BHF Glasgow Cardiovascular Research Centre, University of Glasgow, as described previously [5, 6, 9]. Male rats were housed under controlled conditions of temperature $\left(21^{\circ} \mathrm{C}\right)$ and light (12 h light-dark cycle), were fed on normal rat diet (rat and mouse no. 1 maintenance diet; Special Diet Services, Edinburgh, UK) and had free access to water. The pups were weaned and sexed after 3 weeks and were housed according to sibling group and sex thereafter.

Isolation of adipocytes Epididymal adipocytes were prepared from WKY and SHRSP rats using the collagenase method as described previously [5].

Preparation of adipocyte lysates Epididymal adipocytes were prepared (20\% cytocrit) in Krebs-Ringer-HEPES (KRH) buffer (25 mmol/1 HEPES pH 7.4, $118 \mathrm{mmol} / \mathrm{l}$ $\mathrm{NaCl}, 5 \mathrm{mmol} / 1 \mathrm{NaHCO}_{3}, 4.7 \mathrm{mmol} / \mathrm{l} \mathrm{KCl}, 1.2 \mathrm{mmol} / \mathrm{l}$ $\mathrm{KH}_{2} \mathrm{PO}_{4}, 1.2 \mathrm{mmol} / 1 \mathrm{MgSO}_{4}, 2.5 \mathrm{mmol} / \mathrm{CaCl}_{2}, 1 \%$ [w/v] bovine serum albumin, $200 \mathrm{nmol} / 1$ adenosine, $2.2 \mathrm{mmol} / \mathrm{l}$ glucose). Cells were incubated, with shaking, at $37^{\circ} \mathrm{C}$ in the presence or absence of insulin $(10 \mathrm{nmol} / \mathrm{l})$ for $15 \mathrm{~min}$. Buffer was removed and cells were resuspended in 2 vol lysis buffer $\left(50 \mathrm{mmol} / 1 \mathrm{Tris}-\mathrm{HCl} \mathrm{pH} 7.4\right.$ at $4^{\circ} \mathrm{C}, 50 \mathrm{mmol} / 1$ $\mathrm{NaF}, 5 \mathrm{mmol} / 1 \mathrm{Na}$ pyrophosphate, $1 \mathrm{mmol} / \mathrm{l} \mathrm{Na}$ orthovanadate, $1 \mathrm{mmol} / 1 \mathrm{EDTA}, 1 \mathrm{mmol} / \mathrm{l} \mathrm{EGTA}, 250 \mathrm{mmol} / \mathrm{l} \mathrm{man}$ nitol, $1 \%[\mathrm{v} / \mathrm{v}]$ Triton $\mathrm{X}-100,1 \mathrm{mmol} / 1$ dithiothreitol, $0.1 \mathrm{mmol} / 1 \mathrm{PMSF}, 0.1 \mathrm{mmol} / \mathrm{l}$ benzamidine, $5 \mu \mathrm{g} / \mathrm{ml}$ soybean trypsin inhibitor). Cells were homogenised by 20 passes in a Dounce homogeniser and the resultant homogenate was subjected to centrifugation $(19,000 \times g, 20 \mathrm{~min}$, $\left.4^{\circ} \mathrm{C}\right)$.

RT-PCR and sequencing of GLUT4 PCR amplification of GLUT4 cDNA with Pfu turbo (Stratagene, Amsterdam, The Netherlands) was carried out with total RNA extracted from epididymal adipose tissue with an RNA extraction kit (Qiagen, Crawley, Sussex, UK). PCR products were cloned into PCR2.1 vector (Invitrogen, Paisley, UK) and GLUT4 DNA was sequenced (MWG Biotech, Milton Keynes, UK).

Subcellular fractionation of adipocytes Cells were fractionated by standard techniques. Briefly, epididymal adipocytes were prepared ( $20 \%$ cytocrit) in KRH buffer. Cells were incubated, with shaking, at $37^{\circ} \mathrm{C}$ in the presence or absence of insulin $(10 \mathrm{nmol} / \mathrm{l})$ for $15 \mathrm{~min}$. Buffer was removed and cells were resuspended in 4 vol HES buffer (20 mmol/1 HEPES pH 7.4, $250 \mathrm{mmol} / 1$ sucrose, $1 \mathrm{mmol} / 1$ EDTA, $0.1 \mathrm{mmol} / 1 \mathrm{PMSF}, 0.1 \mathrm{mmol} / 1$ benzamidine, $5 \mu \mathrm{g} / \mathrm{ml}$ 
aprotinin, $1 \mu \mathrm{g} / \mathrm{ml}$ leupeptin). Cells were homogenised by 20 passes in a Dounce homogeniser and the resultant homogenate was subjected to centrifugation $(19,000 \times g$, $20 \mathrm{~min}, 4^{\circ} \mathrm{C}$ ). The $19,000 \times g$ pellet was resuspended, loaded onto a sucrose cushion $(1.12 \mathrm{~mol} / 1$ sucrose, 20 mmol/1 HEPES, pH 7.4, 1 mmol/1 EDTA) and centrifuged $\left(100,000 \times g, 1 \mathrm{~h}, 4^{\circ} \mathrm{C}\right)$. The fraction at the interface was isolated and centrifuged $\left(180,000 \times g, 1 \mathrm{~h}, 4^{\circ} \mathrm{C}\right)$ yielding the plasma membrane (PM) fraction. The supernatant from the $19,000 \times g$ spin was centrifuged $\left(41,000 \times g, 20 \mathrm{~min}, 4^{\circ} \mathrm{C}\right)$ and the subsequent supernatant recentrifuged $(180,000 \times g$, $1 \mathrm{~h}, 4^{\circ} \mathrm{C}$ ). The pellet corresponded to the low-density microsome (LDM) fraction. Fractions were resuspended in HES, and protein concentration was determined using the method of Bradford and stored at $-20^{\circ} \mathrm{C}$ [10].

Triton X-100 solubilisation and sucrose gradient centrifugation of plasma membranes PM pellets were solubilised

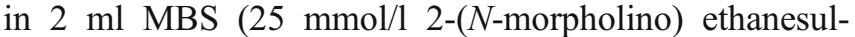
phoric acid [MES] pH 6.5, $150 \mathrm{mmol} / 1 \mathrm{NaCl}, 0.1 \mathrm{mmol} / 1$ PMSF, $0.1 \mathrm{mmol} / \mathrm{l}$ benzamidine, $5 \mu \mathrm{g} / \mathrm{ml}$ aprotinin, $1 \mu \mathrm{g} /$ $\mathrm{ml}$ leupeptin) supplemented with $0.5 \%$ (v/v) Triton X-100. The samples were incubated at $4^{\circ} \mathrm{C}$ for $20 \mathrm{~min}$ with endover-end rotation. Solubilised membranes were homogenised by ten passes in a Dounce homogeniser and added to 1 vol MBS supplemented with $80 \%(\mathrm{w} / \mathrm{v})$ sucrose. The solubilised membranes (in 40\% sucrose) were placed in the bottom of a centrifuge tube and overlaid successively with $6 \mathrm{ml}$ MBS supplemented with $30 \%(\mathrm{w} / \mathrm{v})$ sucrose and $3 \mathrm{ml}$ MBS supplemented with (w/v) 5\% sucrose. After centrifugation $\left(240,000 \times \mathrm{g}, 18 \mathrm{~h}, 4^{\circ} \mathrm{C}\right), 1 \mathrm{ml}$ fractions were collected from the top of the gradient (designated fractions number 1 [top] through 13 [bottom]) and immediately supplemented with protease inhibitors. Equal volumes of all recovered fractions were separated by SDS-PAGE and transferred to nitrocellulose for immunoblotting analysis.

Photolabelling of GLUT4 Epididymal adipocytes were prepared (30\% cytocrit) in KRH buffer and incubated, with shaking, at $37^{\circ} \mathrm{C}$ in the presence or absence of insulin $(1$ or $10 \mathrm{nmol} / \mathrm{l})$ for $15 \mathrm{~min}$. Two aliquots $(100 \mu \mathrm{l})$ from each incubation were spotted onto the wells of a 12-well tissue culture plate and $10 \mu \mathrm{l} 2 \mathrm{mmol} / \mathrm{l}$ Bio-LC-ATBBGPA was added to each. The plate was irradiated for 2 min in a Rayonet UV photochemical reactor and the cells were transferred to microcentrifuge tubes and washed by floatation with $3 \times 1 \mathrm{ml} \mathrm{KRH}$. Cells were lysed in $200 \mu \mathrm{l}$ PBS containing 2\% (w/v) Thesit and $0.1 \mathrm{mmol} / \mathrm{l} \mathrm{PMSF}$, $20 \mu \mathrm{l}$ was stored at $-20^{\circ} \mathrm{C}$ for analysis of total GLUT4 by western blotting and the remainder was centrifuged $\left(15,000 \times \mathrm{g}, 10 \mathrm{~min}, 4^{\circ} \mathrm{C}\right)$. The infranatants were added to $25 \mu \mathrm{l} 50 \%$ streptavidin-agarose and mixed at $4{ }^{\circ} \mathrm{C}$ for $1 \mathrm{~h}$ $30 \mathrm{~min}$, and the pellets were washed $(5 \times 1 \mathrm{ml} \mathrm{PBS}+1 \%$ Thesit) prior to storage at $-20^{\circ} \mathrm{C}$.

Electrophoresis and immunoblotting Proteins were electrophoresed on SDS polyacrylamide gels and transblotted onto nitrocellulose membranes as described previously $[5,6]$. Immunolabelled proteins were visualised using horseradish peroxidase-conjugated secondary antibody and the enhanced chemiluminescence system (Amersham Biosciences).

Statistics Unless otherwise stated, results are expressed as means \pm SD. Statistically significant differences were determined using a two-tailed independent-samples Student's $t$-test, and a $p$ value of less than 0.05 was considered significant.

\section{Results}

We have previously demonstrated that epididymal adipocytes and skeletal muscles isolated from SHRSP animals exhibit reduced insulin-stimulated glucose uptake $[5,6]$. The glucose transporter GLUT4 mediates insulin-stimulated glucose transport in adipocytes and skeletal muscle $[1,2]$. We therefore examined the ability of insulin to stimulate the translocation of GLUT4 from intracellular membranes to the plasma membrane by determining the distribution of GLUT4 after subcellular fractionation of adipocytes from SHRSP and WKY animals incubated in the presence or absence of insulin. GLUT4 exhibited translocation to the plasma membrane in response to acute in-

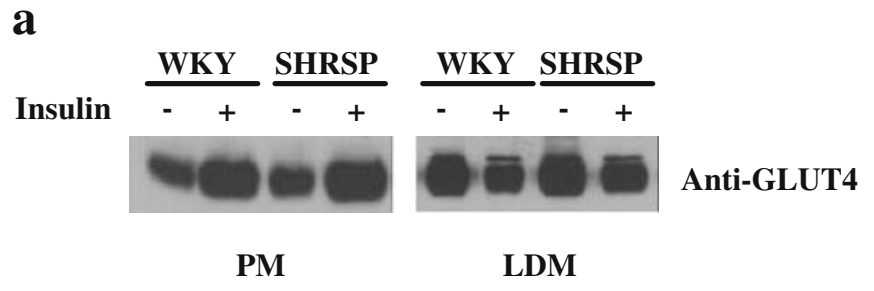

b
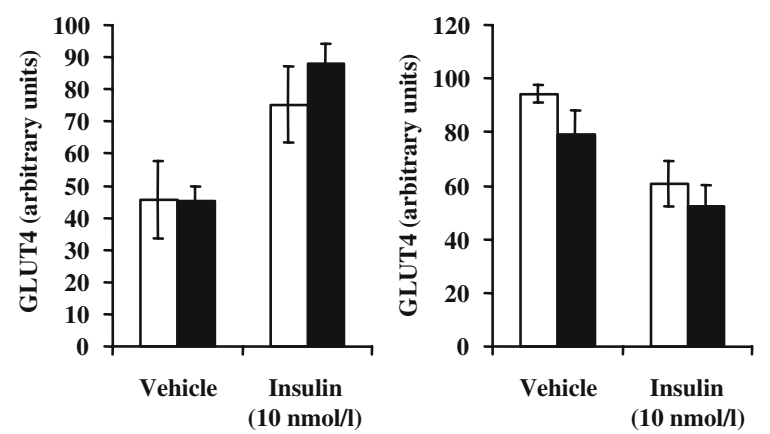

PM

LDM

Fig. 1 The effect of insulin on subcellular distribution of GLUT4 in rat epididymal adipocytes. Epididymal adipocytes were isolated from WKY rats (open bars) and SHRSP rats (filled bars) and incubated in the presence or absence of insulin $(10 \mathrm{nmol} / \mathrm{l})$ for 15 min. Cells were homogenised and plasma membrane $(P M)$ and low-density microsome $(L D M)$ fractions prepared as described in the text. SDS-PAGE was performed using equal quantities $(10 \mu \mathrm{g})$ from each fraction. The distribution of GLUT4 was then studied by immunoblotting. a Data from a single representative experiment b Quantification of data from six independent fractionation experiments of this type (presented as arbitrary intensity units, means \pm SD) 
sulin treatment in both WKY and SHRSP animals (Fig. 1). Insulin stimulated GLUT4 translocation 1.7-fold $(p<0.05)$ and 1.9 -fold $(p<0.005)$ in adipocyte plasma membranes derived from WKY and SHRSP animals, respectively. Conversely, stimulation with insulin resulted in a reduction of GLUT4 in adipocyte LDM fractions of $35 \%(p<0.05)$ and $34 \%(p<0.05)$ from WKY and SHRSP animals respectively. However, there was no significant difference in GLUT4 expression in PM or LDM fractions obtained under basal or insulin-stimulated conditions when comparing the two rat strains. The trafficking of insulin-regulated aminopeptidase (IRAP) and the v-SNARE vesicle-associated membrane protein-2 (VAMP2) has been shown to be identical to that of GLUT4 [11, 12]. We therefore assessed trafficking of IRAP (Fig. 2) and VAMP2 (Fig. 3) by western blotting of subcellular fractions obtained from SHRSP and WKY animals incubated in the presence or absence of insulin. IRAP exhibited translocation to the plasma membrane in response to acute insulin treatment in both WKY and SHRSP animals (Fig. 2). Insulin stimulated IRAP translocation 2.6-fold $(p<0.05)$ and 2.1-fold $(p<0.05)$ in adipocyte plasma membranes derived from WKY and SHRSP animals, respectively. Conversely, stimulation with insulin resulted in a reduction of IRAP in adipocyte LDM fractions

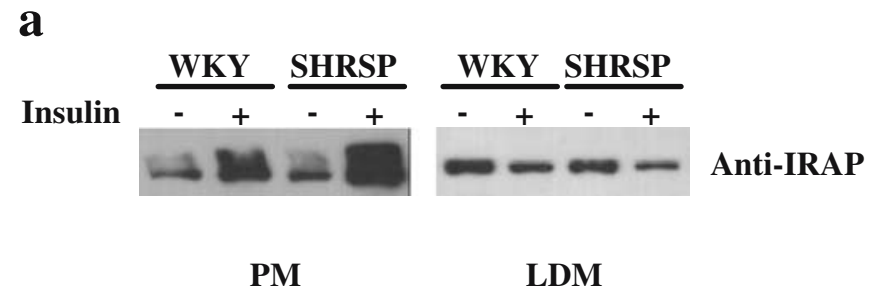

b

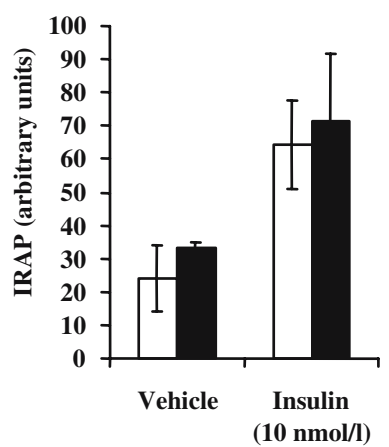

PM

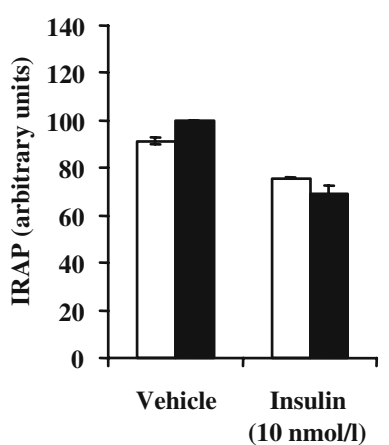

LDM
Fig. 2 The effect of insulin on subcellular distribution of IRAP in rat epididymal adipocytes. Epididymal adipocytes were isolated from WKY rats (open bars) and SHRSP rats (filled bars) and incubated in the presence or absence of insulin $(10 \mathrm{nmol} / \mathrm{l})$ for $15 \mathrm{~min}$. Cells were homogenised and plasma membrane $(P M)$ and low-density microsome $(L D M)$ fractions prepared as described in the text. SDS-PAGE was performed using equal quantities $(10 \mu \mathrm{g})$ from each fraction. The distribution of IRAP was then studied by immunoblotting. a Data from a single representative experiment. b Quantification of data from four independent fractionation experiments of this type (presented as arbitrary intensity units, means $\pm \mathrm{SD}$ ) $\mathbf{a}$

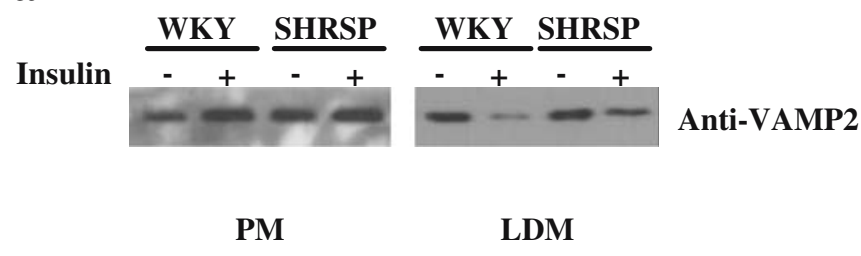

b
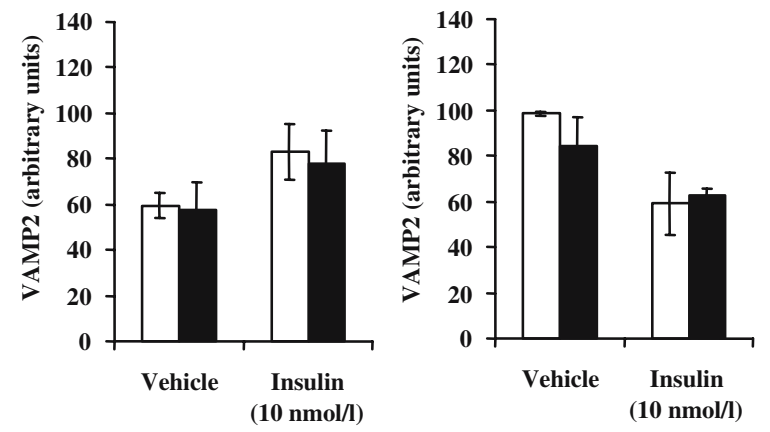

PM

LDM

Fig. 3 The effect of insulin on subcellular distribution of VAMP2 in rat epididymal adipocytes. Epididymal adipocytes were isolated from WKY rats (open bars) and SHRSP rats (filled bars) and incubated in the presence or absence of insulin $(10 \mathrm{nmol} / \mathrm{l})$ for 15 min. Cells were homogenised and plasma membrane $(P M)$ and low-density microsome $(L D M)$ fractions prepared as described in the text. SDS-PAGE was performed using equal quantities $(10 \mu \mathrm{g})$ from each fraction. The distribution of VAMP2 was then studied by immunoblotting. a Data from a single representative experiment. b Quantification of data from four independent fractionation experiments of this type (presented as arbitrary intensity units, means \pm SD)

of $18 \%(p<0.05)$ and $31 \%(p<0.05)$ from WKY and SHRSP animals respectively. However, there was no significant difference in IRAP expression in PM or LDM fractions obtained under basal or insulin-stimulated conditions when comparing the two rat strains. VAMP2 exhibited no significant translocation to the plasma membrane in response to acute insulin treatment in either WKY or SHRSP animals (Fig. 3). However, stimulation with insulin resulted in a reduction of VAMP2 in adipocyte LDM fractions of 40\% $(p<0.05)$ and 26\% $(p<0.05)$ from WKY and SHRSP animals, respectively. There was no significant difference in VAMP2 expression in PM or LDM fractions obtained under basal or insulin-stimulated conditions when comparing the two rat strains.

We next determined if the reduction in insulin-stimulated glucose transport was the result of a mutation in the coding sequence of GLUT4. Sequencing of GLUT4 cloned from cDNA derived from SHRSP epididymal adipose demonstrated that the coding sequence of GLUT4 was not different from that of the WKY control rats (data not shown).

Using a glucose photolabel (Bio-LC-ATB-BGPA) to tag cell surface GLUT4, we then determined the levels of GLUT4 available to substrate at the plasma membrane of adipocytes from WKY and SHRSP rats after stimulation with insulin. GLUT4 exhibited translocation to the cell sur- 
face in response to acute insulin treatment in both WKY and SHRSP animals in a dose-dependent manner (Fig. 4). After insulin stimulation $(10 \mathrm{nmol} / \mathrm{l})$, cell surface GLUT4 was significantly attenuated in SHRSP adipocytes $(56 \%$ of cell surface GLUT4 in WKY adipocytes, $p<0.05$ ). There was no significant difference in the total cellular expression of GLUT4 between the rat strains as illustrated in Fig. 4.

In muscle, discrepancies have been observed between the magnitude of GLUT4 translocation and glucose transport and it has been proposed that there is an increase in the intrinsic activity of GLUT4 in addition to its translocation [13]. Furthermore, insulin stimulation of p38MAPK has been proposed to account for this increase in intrinsic activity [12]. We therefore examined the effects of insulin on p38MAPK activity using an antibody that recognises doubly phosphorylated (T180 and Y182) p38MAPK (Fig. 5a). Phosphorylation at these sites is necessary for activation of p38MAPK. Western blotting of lysates prepared from SHRSP and WKY adipocytes incubated in the presence or absence of insulin revealed no significant alteration in p38MAPK expression in SHRSP compared with WKY (Fig. 5b). In addition, incubation with insulin had no significant effect on the amount of phosphorylated p38MAPK in either rat strain (Fig. 5c). The levels of phosphorylated p38MAPK were also not significantly different between the strains.

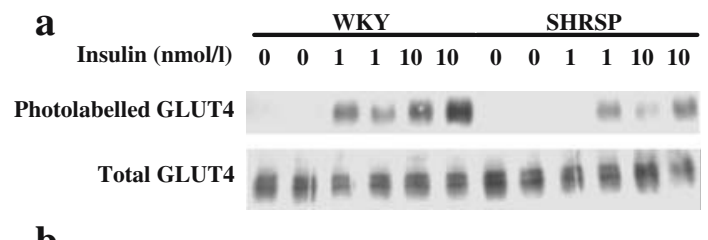

b

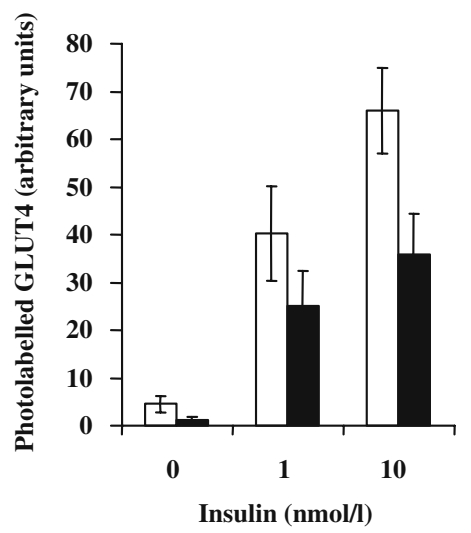

Fig. 4 Insulin-stimulated GLUT4 translocation to the cell surface is attenuated in SHRSP adipocytes compared with in WKY controls. Epididymal adipocytes were isolated from WKY rats (open bars) and SHRSP rats (filled bars) and incubated in the presence or absence of insulin (1 or $10 \mathrm{nmol} / \mathrm{l}$ ) for $15 \mathrm{~min}$. Cell surface GLUT4 was tagged with the biotinylated photolabel Bio-LC-ATB-BGPA and separated using streptavidin-agarose as described in the text. SDS-PAGE was performed on both streptavidin-agarose-precipitated proteins and whole cell lysate. The levels of GLUT4 were then studied by immunoblotting. a Data from a single representative experiment. b Quantification of data from five independent fractionation experiments of this type, performed in duplicate (presented as arbitrary intensity units, means $\pm \mathrm{SD}$ )
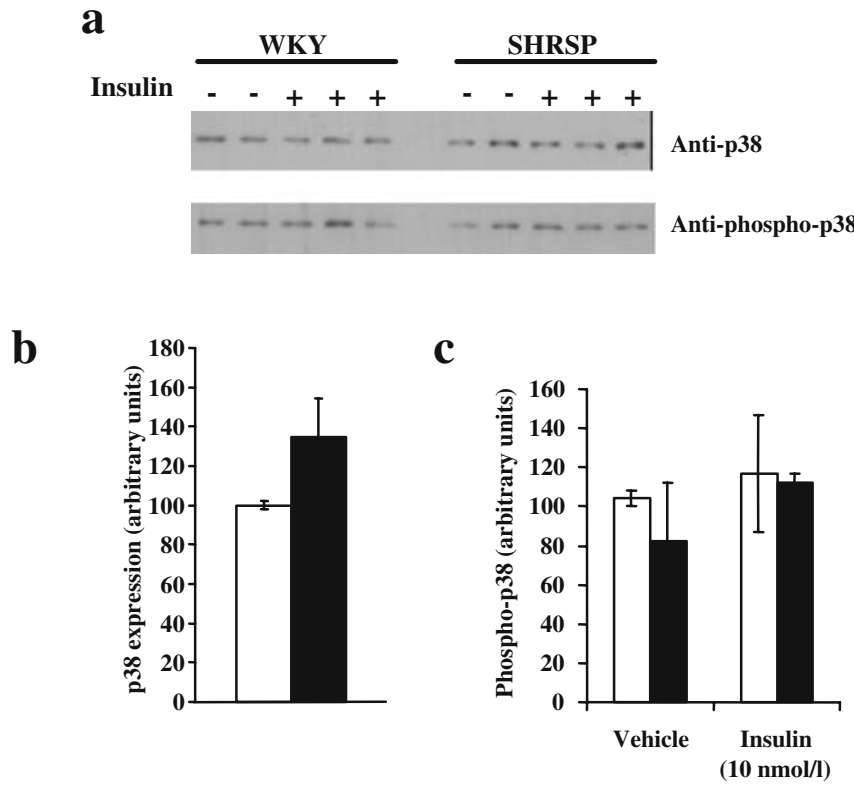

Fig. 5 The effect of insulin on $p 38 M A P K$ phosphorylation in rat epididymal adipocytes. Epididymal adipocytes were isolated from WKY rats (open bars) and SHRSP rats (filled bars) and incubated in the presence or absence of insulin $(10 \mathrm{nmol} / \mathrm{l})$ for $15 \mathrm{~min}$. Cell lysates were prepared and SDS-PAGE was performed using equal quantities $(10 \mu \mathrm{g})$ of protein. The expression of $\mathrm{p} 38 \mathrm{MAPK}$ and extent of p38MAPK phosphorylation were assessed by immunoblotting. a Data from a single representative experiment. b Quantification of p38 expression. c p38 phosphorylation from three independent experiments (presented as arbitrary intensity units, means \pm SD)

It has previously been shown that insulin stimulates GLUT4 translocation to the PM, followed by transition of GLUT4 to a caveolin-rich plasma membrane lipid raft domain in rat epididymal adipocytes [14]. We therefore deter-

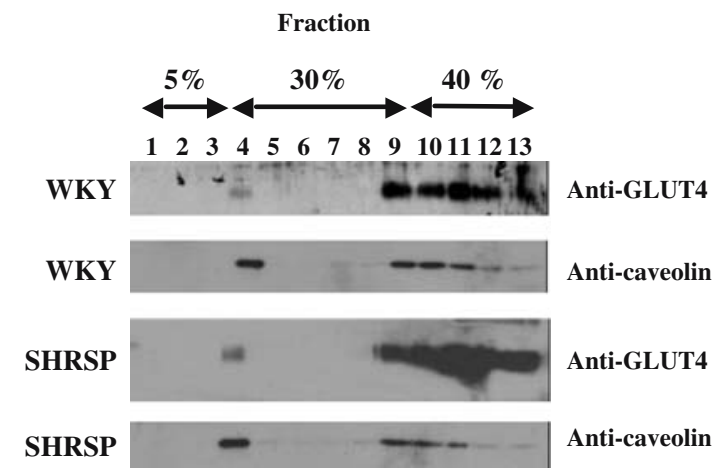

Fig. 6 The effect of insulin on GLUT4 association with caveolinrich domains in rat epididymal adipocytes plasma membrane fractions. Epididymal adipocytes were isolated from $W K Y$ and $S H R S P$ rats and incubated in the presence of insulin $(10 \mathrm{nmol} / \mathrm{l})$ for $15 \mathrm{~min}$. Cells were homogenised and PM pellets prepared, solubilised and subjected to discontinuous sucrose gradient centrifugation as described in the text. Fractions were collected from the top of the gradient (fraction 1 is the top fraction) and SDS-PAGE was performed using equal volumes $(20 \mu \mathrm{l})$ from each fraction. The distribution of GLUT4 and caveolin was then studied by immunoblotting using the antibodies indicated. Immunoblots from a single representative experiment are shown 
mined the proportion of PM GLUT4 in caveolin-rich lipid raft fractions after insulin stimulation of SHRSP and WKY adipocytes. This procedure separates solubilised proteins from insoluble lipid rafts, as shown by the separation of caveolin (a marker protein for rafts) (Fig. 6). Solubilised proteins remain in the $40 \%$ sucrose layer, whereas insoluble rafts accumulate at the interface of the $5 \%$ and $30 \%$ sucrose layers. Western blotting of discontinuous sucrose gradient fractions prepared from WKY and SHRSP plasma membranes incubated in the presence of insulin revealed that a small proportion $(4 \pm 1 \%$ and $4 \pm 2 \%$ respectively, $n=3)$ of total PM GLUT4 is present in caveolin-rich fractions (Fig. 6). There was no significant difference in distribution between the strains. The expression and distribution of caveolin in adipocyte PM fractions was also not significantly different between the strains (data not shown).

\section{Discussion}

Insulin resistance is associated with a number of metabolic and haemostatic abnormalities including hypertension [15]. We have recently demonstrated that the SHRSP, which is considered to be a good model for human essential hypertension, exhibits impaired insulin action in isolated adipocytes and skeletal muscles $[5,6]$. In this study we have determined the effect of insulin on GLUT4 translocation to the plasma membrane in isolated adipocytes from SHRSP and WKY. The most significant findings of this study were that the magnitude of insulin-stimulated trafficking of GLUT4 from intracellular membranes to the plasma membrane was not significantly different between SHRSP and WKY animals, yet SHRSP animals exhibited markedly reduced GLUT4 available to a glucose photolabel compared with WKY controls. We propose that this reduction in bioavailable GLUT4 underlies impaired insulin-stimulated glucose transport in adipocytes derived from SHRSP animals.

The magnitude of insulin-stimulated GLUT4, IRAP and VAMP2 translocation from LDM to PM fractions is quantitatively similar to that previously demonstrated in 3T3-L1 adipocytes $[7,16,17]$. The lack of any significant difference in insulin-stimulated GLUT4 translocation (in addition to IRAP and VAMP2) between the rat strains indicates that the markedly attenuated insulin-stimulated glucose transport we have demonstrated previously in SHRSP adipocytes [5] is not due to reduced trafficking of GLUT4 to the plasma membrane. In addition, the reduced insulin-stimulated glucose transport is not a result of altered GLUT4 protein expression or variant GLUT4 protein sequence.

Using the photolabel Bio-LC-ATB-BGPA we demonstrated that markedly reduced GLUT4 at the plasma membrane after insulin stimulation was available to substrate in SHRSP compared with WKY rats. This implies that a large proportion of GLUT4 translocated to the plasma membrane in response to insulin is not able to bind substrate and catalyse transport in the SHRSP. Therefore, there is a reduction in bioavailable GLUT4 in SHRSP animals that is likely to account, at least in part, for the reduced insulinstimulated glucose uptake.

It has been proposed that discrepancies between the magnitude of insulin-stimulated glucose transport and GLUT4 translocation demonstrated in adipocytes and muscle are the result of insulin stimulating both the translocation of GLUT4 and increasing the intrinsic activity of the transporters [13]. Furthermore, p38MAPK has been proposed as a candidate that mediates insulin stimulation of GLUT4 intrinsic activity [13]. In 3T3-L1 adipocytes, insulin has been demonstrated to stimulate p38MAPK phosphorylation (a measure of activity) [18, 19]. However, insulin had no effect on p38MAPK phosphorylation in human adipocytes from healthy subjects or patients with type 2 diabetes [18]. Insulin had no effect on p38MAPK phosphorylation in either rat strain in the current study. The discrepancies between these studies are likely to be a result of the different tissues used, and in this respect 3T3-L1 adipocytes may not reflect the signalling pathways utilised in either human or rat isolated adipocytes. We cannot, therefore, rule out impaired insulin-stimulated increases in GLUT4 intrinsic activity as a mechanism behind reduced insulin-stimulated glucose transport in the SHRSP animals. It is unlikely, however, that aberrant insulin-stimulated p38MAPK activity underlies this. Increased expression of p38MAPK has been reported in adipocytes from patients with type 2 diabetes compared with healthy controls [18] Although there was a tendency for increased p38MAPK expression in SHRSP animals, this did not reach significance in the current study.

It has been demonstrated that GLUT4 is partially localised in lipid rafts in both rat epididymal adipocytes and cultured 3T3-L1 adipocytes [14, 20]. A small proportion of the total GLUT4 in plasma membranes is localised to caveolin-rich lipid raft domains in both rat strains in the current study after insulin stimulation. The lack of significant difference in the proportion of total GLUT4 in these fractions between the rat strains indicates that the reduced insulin-stimulated glucose transport in SHRSP adipocytes is not due to altered association of GLUT4 with such lipid raft domains.

An alternative explanation for the reduced insulin-stimulated glucose transport in the SHRSP is that a large proportion of GLUT4 translocated to the plasma membrane in response to insulin is not inserted appropriately in the membrane, or is in vesicles that are docked with the plasma membrane but have not fused efficiently or appropriately with it. Such vesicles would be indistinguishable from the plasma membrane as assessed by western blotting after subcellular fractionation, but might well be inaccessible to photolabel.

Intriguingly, adipocytes derived from SHRSP share common features with adipocytes derived from rats subjected to long-term ethanol feeding [21,22]. Epididymal adipocytes from ethanol-fed rats exhibit markedly reduced insulin-stimulated glucose transport and reduced insulinstimulated surface accessibility of GLUT4 to a photolabel similar to that used in this study, whereas there was only 
a small reduction in insulin-stimulated translocation of GLUT4 to the plasma membrane [22]. Furthermore, activation of adenosine $A_{1}$ receptors increased glucose transport in adipocytes from both ethanol-fed and control rats [22]. The authors of that study argued that ethanol-induced increases in stimulatory heterotrimeric G-proteins $\left(\mathrm{G}_{\mathrm{s}}\right)$ relative to inhibitory heterotrimeric G-proteins $\left(\mathrm{G}_{\mathrm{i}}\right)$ contribute to the decreased ability of insulin to stimulate glucose transport in these cells [22]. In the current study, adipocytes were isolated and manipulated in the presence of $200 \mathrm{nmol} / \mathrm{l}$ adenosine. As $\mathrm{G}_{\mathrm{i}}$-linked $\mathrm{A}_{1}$ receptors represent the major adenosine receptor subtype present in adipocytes, it seems unlikely that reduced stimulation of $\mathrm{G}_{\mathrm{i}}$ would have occurred in the present study unless the $\mathrm{G}_{\mathrm{i}}$ system is aberrant in SHRSP adipocytes. The $\mathrm{G}_{\mathrm{s}}$ and $\mathrm{G}_{\mathrm{i}}$ signalling systems have yet to be characterised in SHRSP adipocytes. Indeed, altered $\mathrm{G}_{\mathrm{s}} / \mathrm{G}_{\mathrm{i}}$ protein expression, defective receptor- $G$ protein coupling or aberrant downstream signalling cannot be discounted as a mechanism that underlies attenuated insulin-stimulated glucose transport in SHRSP adipocytes and further investigations are required.

Isoproterenol has been demonstrated to inhibit insulinstimulated glucose transport and exposure of GLUT4 to photolabel in rat adipocytes without altering GLUT4 expression at the plasma membrane [23]. The effects of isoproterenol on insulin-stimulated glucose transport were mimicked by acidification and reversed by alkalinisation of the adipocytes, suggesting the effects of insulin are $\mathrm{pH}$ sensitive [24]. Insulin has been proposed to stimulate alkalinisation by stimulation of $\mathrm{Na}^{+} / \mathrm{H}^{+}$exchange in erythrocytes and myocytes $[24,25]$. However, $\mathrm{Na}^{+} / \mathrm{H}^{+}$exchange has been shown to be elevated in vascular smooth muscle cells derived from SHRSP [26], arguing against a role for reduced cell alkalinisation in aberrant insulin-stimulated GLUT4 translocation in adipocytes. Further studies are required, however, to determine whether a perturbation in intracellular $\mathrm{pH}$ explains the aberrant insulin-stimulated glucose transport in the SHRSP adipocytes.

Insulin-stimulated translocation of GLUT4 to the plasma membrane is a highly regulated process. The fidelity of this process is mediated at least in part by SNARE proteins [27]. In adipocytes and muscle, the SNAREs syntaxin 4 and VAMP2 have been identified as being of crucial importance in insulin-stimulated GLUT4 translocation [7, $12,28,29]$. We have previously demonstrated that expression of syntaxin 4 and VAMP2 is increased in muscles derived from SHRSP animals compared with in WKY animals [6], in addition to in the Zucker diabetic fatty rat [30]. In the current study, there was no significant difference in the expression of VAMP2 in PM and LDM fractions between the strains, but this does not preclude increased total VAMP2 expression in SHRSP adipocytes. It remains to be determined whether the upregulation of SNAREs in muscle is a causal or adaptive mechanism in the pathogenesis of insulin resistance in the SHRSP. Further studies to determine whether there is increased expression of SNAREs in SHRSP adipocytes relative to WKY are required.
In conclusion, there is reduced bioavailable GLUT4 present in the plasma membrane of SHRSP adipocytes compared with in WKY control animals. This impairment is likely to underlie the reduced insulin-stimulated glucose transport exhibited in adipocytes from SHRSP animals. Further studies are required to determine the molecular mechanism(s) by which this impairment is manifest.

Acknowledgements This work was supported by the British Heart Foundation (Fellowship to I.P. Salt and Programme Grant and Chair to A.F. Dominiczak) and Medical Research Council (Cooperative Group Grant to J.M.C. Connell, A.F. Dominiczak and G.W. Gould).

\section{References}

1. Elmendorf JS (2002) Signals that regulate GLUT4 translocation. J Memb Biol 190:167-174

2. Saltiel AR, Kahn CR (2001) Insulin signalling and the regulation of glucose and lipid metabolism. Nature 414:799-805

3. Aitman TJ, Glazier AM, Wallace CA et al (1999) Identification of Cd36 (Fat) as an insulin-resistance gene causing defective fatty acid and glucose metabolism in hypertensive rats. Nat Genet 21:76-83

4. Reaven GM, Chang H, Hoffmann BB, Azhar S (1989) Resistance to insulin-stimulated glucose uptake in adipocytes isolated from spontaneously hypertensive rats. Diabetes 38: $1155-1160$

5. Collison M, Glazier AM, Aitman TJ et al (2000) Cd36 and molecular mechanisms on insulin resistance in the stroke-prone spontaneously hypertensive rat. Diabetes 49:2222-2226

6. James DJ, Cairns F, Salt IP et al (2001) Skeletal muscle of stroke-prone spontaneously hypertensive rats exhibits reduced insulin-stimulated glucose transport and elevated levels of caveolin and flotillin. Diabetes 50:2148-2156

7. Millar CA, Sherwan A, Hickson GRX, James DE, Gould GW (1999) Differential regulation of secretory components containing the insulin-responsive glucose transporter, GLUT4 in 3T3L1 adipocytes. Mol Biol Cell 10:3675-3688

8. Hashimoto M, Hatanaka Y, Yang J, Dhesi J, Holman GD (2001) Synthesis of biotinylated bis(D-glucose) derivatives for glucose transporter photoaffinity labelling. Carbohydr Res 331: $119-127$

9. Davidson AO, Schork N, Jaques BC et al (1995) Blood pressure in genetically hypertensive rats. Hypertension 26:452-459

10. Bradford MM (1976) A rapid and sensitive method for the quantitation of microgram quantities of protein utilizing the principle of protein-dye binding. Anal Biochem 72:248-254

11. Garza LA, Birnbaum MJ (2000) Insulin-responsive aminopeptidase trafficking in 3T3-L1 adipocytes. J Biol Chem 275: $2560-2567$

12. Martin LB, Shewan A, Millar CA, Gould GW, James DE (1998) Vesicle-associated membrane protein 2 plays a specific role in the insulin-dependent trafficking of the facilitative glucose transporter GLUT4 in 3T3-L1 adipocytes. J Biol Chem 273:1444-1452

13. Furtado LM, Poon V, Klip A (2003) GLUT4 activation: thoughts on possible mechanisms. Acta Physiol Scand 178:287-296

14. Gustavsson J, Parpal S, Strålfors P (1996) Insulin-stimulated glucose uptake involves the transition of glucose transporters to a caveolae-rich fraction within the plasma membrane: implications for type II diabetes. Mol Med 2:367-372

15. Laight DW, Carrier MJ, Anggard EE (2000) Antioxidants, diabetes and endothelial dysfunction. Cardiovasc Res 47:457464

16. Chamberlain LH, Gould GW (2002) The vesicle- and targetSNARE proteins that mediate Glut4 vesicle fusion are localised in detergent-insoluble lipid rafts present on distinct intracellular membranes. J Biol Chem 277:49750-49754 
17. Perera HKI, Clarke M, Morris NJ, Hong W, Chamberlain LH, Gould GW (2003) Syntaxin 6 regulates Glut4 trafficking in 3T3-L1 adipocytes. Mol Biol Cell 14:2946-2958

18. Carlson CJ, Koterski S, Sciotti RJ, Braillard Poccard G, Rondinone CM (2003) Enhanced basal activation of mitogenactivated protein kinases in adipocytes from type 2 diabetes. Diabetes 52:634-641

19. Sweeney G, Somwar R, Ramlal T, Volchuk A, Ueyama A, Klip A (1999) An inhibitor of p38 mitogen-activated protein kinase prevents insulin-stimulated glucose transport but not glucose transporter translocation in 3T3-L1 adipocytes and L6 myotubes. J Biol Chem 274:10071-10078

20. Ros-Baró A, López-Iglesias C, Peiró S et al (2001) Lipid rafts are required for GLUT4 internalization in adipose cells. Proc Natl Acad Sci U S A 98:12050-12055

21. Wilkes JJ, Deforrest LL, Nagy LE (1996) Chronic ethanol feeding in a high-fat diet decreases insulin-stimulated glucose transport in rat adipocytes. Am J Physiol 271:E477-E484

22. Poirier LA, Rachdaoui N, Nagy LE (2001) GLUT4 vesicle trafficking in rat adipocytes after ethanol feeding: regulation by heterotrimeric G-proteins. Biochem J 354:323-330

23. Yang J, Hodel A, Holman GD (2002) Insulin and isoproterenol have opposing roles in the maintenance of cytosol $\mathrm{pH}$ and optimal fusion of GLUT4 vesicles with the plasma membrane. J Biol Chem 277:6559-6566

24. Incerpi S, Rizvi SI, De Vito P, Luly P (1997) Insulin stimulation of $\mathrm{Na} / \mathrm{H}$ antiport in L-6 cells: a different mechanism in myoblasts and myotubes. J Cell Physiol 171:235-242
25. Sauvage M, Maziere P, Fathallah H, Giraud F (2000) Insulin stimulates NHE1 activity by sequential activation of phosphatidylinositol 3-kinase and protein kinase $\mathrm{C}$ zeta in human erythrocytes. Eur J Biochem 267:955-962

26. Orlov SN, Adarichev VA, Devlin AM et al (2000) Increased $\mathrm{Na}^{+} / \mathrm{H}^{+}$exchanger isoform 1 activity in spontaneously hypertensive rats: lack of mutations within the coding region of NHE1. Biochim Biophys Acta 1500:169-180

27. Pessin JE, Thurmond DC, Elmendorf JS, Coker KJ, Okada S (1999) Molecular basis of insulin stimulated GLUT4 vesicle trafficking: location! location! location! J Biol Chem 274:25932596

28. Cheatham B, Volchuk A, Katin CR, Wang L, Rhodes C, Klip A (1996) Insulin-stimulated translocation of GLUT4 glucose transporters requires SNARE-complex proteins. Proc Natl Acad Sci U S A 93:15169-15173

29. Volchuk A, Wang Q, Ewart HS et al (1996) Syntaxin 4 in 3T3L1 adipocytes: regulation by insulin and participation in insulin-dependent glucose transport. Mol Biol Cell 7:1075-1082

30. Maier V, Melvin DR, Lister CA, Chapman H, Gould GW, Murphy GJ (2000) v- and t-SNARE protein expression in models of insulin-resistance: normalisation of glycaemia by rosiglitazone treatment corrects overexpression of cellubrevin, vesicle-associated membrane protein-2, and syntaxin 4 in skeletal muscle of Zucker diabetic fatty rats. Diabetes 49:618-625 